\title{
The Mirror Strategy of Nanoparticles Against the Coronavirus.
}

\author{
$\underline{\text { Zahra Fakhroueian }}^{\mathrm{a}^{*}}$, Pegah Esmaeilzadeh $^{\mathrm{b}}$, Pouriya Esmaeilzadeh $^{\mathrm{c}}$
}

\begin{abstract}
${ }^{a}$ School of Chemical Engineering-Nanomedicine, College of Engineering, Institute of Petroleum Engineering (IPE), University of Tehran, Iran. ${ }^{\mathrm{b}}$ Institute of Pharmacy, Martin Luther University Halle Wittenberg, Heinrich Damerow Strasse 4, 06120 Halle (Saale), Germany. ${ }^{\mathrm{c}}$ Process simulation and control Lab., School of Chemical Engineering-Nanoengineering, Iran University of Science and Technology (IUST), Narmak, Tehran, Iran.

*Corresponding author: Zahra Fakhroueian, PhD, Post doc in nanomedicine, University of Tehran Iran.
\end{abstract}

Email: $\underline{\text { fakhroueian@ut.ac.ir }}$

Fax: +982188632976

\begin{abstract}
:
Research on the chemical mechanism and reciprocal behavior of the coronavirus relate to living organisms, engaging in the give and take of electrochemical mediators, is a very important, controversial and vital issue. What we should accept is the chemical identity of this scenario, and not preferably a characteristic of a biological system. This chemical reaction should be familiar, referring to the theory of chemical pathways involved in DNA/proteins in the body against aggressive guests (such as viruses). From the point of view of a chemist, this simple reaction is nothing more than an oxidation-reduction reaction (redox-stress signaling) which conducted and carried out by coronavirus in a biointerface medium. Thereby, oxidizing as well as reducing reagents should be very constructive, promoting development in such chemical process. We understand redox reactions as switchable thiol/disulfide exchanges (formation and cleavage of inherent disulfide bonds), then, we can hugely profit from redox-responsive nano-surfaces equipped with multiple new ionic and covalent interactions. This game-changing idea can substantiate by surface modified-nanoparticles to play powerful roles in synthesis of nano oxidizers as well as reducing agents in nanomedicine. Chemists and pharmacists must then explore new thoughts and present modern experiences/approaches of preparation nanoparticles and nanocomposites to create novel vaccines as well as coronavirus drugs. In this regard, this experience can also be so helpful for HIV/AIDS, which is caused by viruses.
\end{abstract}

Keywords: Coronavirus Disease 2019, Covid-19 Pandemic, HIV/AIDS, Nanoantivirals, Smart Nanomaterials, Synergistic Oxidant @ Reductant Agents, Antiviral Coatings, Oxidizer and Reductant Nano Agents, Nanomedicine.

\section{New ideas in covid-19 by chemists:}


(a): To achieve the long-term goals in the future, trust the new and advanced knowledge of nanotechnology and nanomedicine.

In this part of proposal, we try to identify the nano-oxidizer and nano-reducing constituents. Many oxidizing nanoparticles (NPs), such as various quantum dot semiconductor NPs (ZnO), iron oxide NPs, and all metal oxide NPs, can play very influential roles in a reversible redox [1]. Oxidizing NPs must be able to gain electrons and initiate oxidation reactions, and then reduce themselves. Strong reducing NPs must be able to give electrons and carry out reduction reaction, then oxidize themselves. Relying on our expertise and knowledge of surface modifications of NPs, as an effective reductant, we suggest carbon nanotubes (CNTs) NPs which their surfaces have been functionalized with $\mathrm{Cu}$ NPs, Ag NPs, Au NPs (but these are not economical enough), or just by functional groups of $-\mathrm{COOH}$ (carboxyl), and $-\mathrm{RCONH}_{2}$ (amide) since they can be reduced to $\mathrm{CO}_{2}$ and aldehydes, respectively.

Herein, CNTs NPs alone and without surface modifications could not be a strong enough reductant to give electrons to protein cell membrane of coronavirus structure. As this is a new experience, for providing more confidence that CNTs NPs will impart reducing role, we suggest to modify its surface. Besides CNTs NPs alone could be toxic and insoluble in water-based solutions, being not an eco-friendly agent. We suggest with - $\mathrm{COOH}$ modification (for example from oleic acid (C18$\mathrm{COOH})$ or soya green fatty acid $(\mathrm{C} 18)$, remove the problem of toxicity and solvation, but more importantly help the CNTs NPs to release/donate electrons to virus' amino acids.

\section{(b): The effect of covid-19 (as a terrible virus) on the body.}

The virus covid-19 has a chemical structure with a protein coating (aminoacid groups) [2-3]. This protein coating is called a capsid and a lipid membrane also surrounds the protein coating. Inside this protein coating are nucleic acids (polymers) with long chains and high molecular weight composed of nucleotides. Each nucleotide consists of the following parts: a phosphoric acid molecule, a C5 sugar molecule, and an organic base containing nitrogen molecule. On the other hand, nucleic acids are single or double strands of DNA or RNA that contain vital virus information. Recently, some of scientists believe that viruses are living organisms due to their DNA and RNA and the growth and proliferation in other organisms as guests. The covid-19 virus, as well as HIV, destroys the human immune system and T cells in the body [4-5]. Knowing that viruses have always been said not to be alive, but with these sudden mutations and changes in the functioning of the human body, it can be imagined that the virus could be a living donor. Giving electrons is an entrance to the topic of reduction reactions and dynamic properties that are kept employing in natural systems. As part of a host-guest chemistry, amino-acids of virus can give some electrons to the host (such as disulfide bond linkage in protein cysteine residues) and reduce them and oxidize itself. Therefore, in proposed chemical process, covid-19 virus (or HIV virus) is a strong reducing agent against disulfides, whereas disulfide bonds are destabilized and broken to thiol radicals in vivo [6]. It is actually possible to consider this theory that a regenerative virus enters the host protein membrane, and tries to damage the folding body of vital proteins, damaging/breaking the $-\mathrm{S}$-S-protein disulfide bonds. The result is generation of sulfhydryls (thiols) 
or their anion radicals [7]. The thiol group represents the "-2 oxidation state" of the sulfur atom. Figure 1 can show this total vital chemical reaction.

$\mathrm{R}-\mathrm{S}-\mathrm{S}-\mathrm{R}+\mathrm{ne}^{-}$( $\mathrm{n}$ : number of electrons originates from coronavirus as a reducing agent)

$$
\mathrm{R}-\mathrm{SH}+\mathrm{SH}-\mathrm{R} \text { (thiol products) }
$$

(The destroyer reduction chemical reaction)

$$
\mathrm{R}-\mathrm{S}-\mathrm{H}+\mathrm{R}-\mathrm{S}-\mathrm{H}+\text { Nanoparticles as a suitable strong oxidant } \longrightarrow \mathrm{R}-\mathrm{S}-\mathrm{S}-\mathrm{R}+2 \mathrm{H}++2 \mathrm{e}^{-}
$$

(The restorative oxidation reaction by nanoparticles)

Thus, viruses play a destructive role in the body's vital proteins, and it can continue to destroy all the body's folding protein structures. Finally, the thiol-disulfide exchange rearrangements would be finished by powerful nanoparticles.

\section{Denaturation by cancer cell, bacteria, and virus}
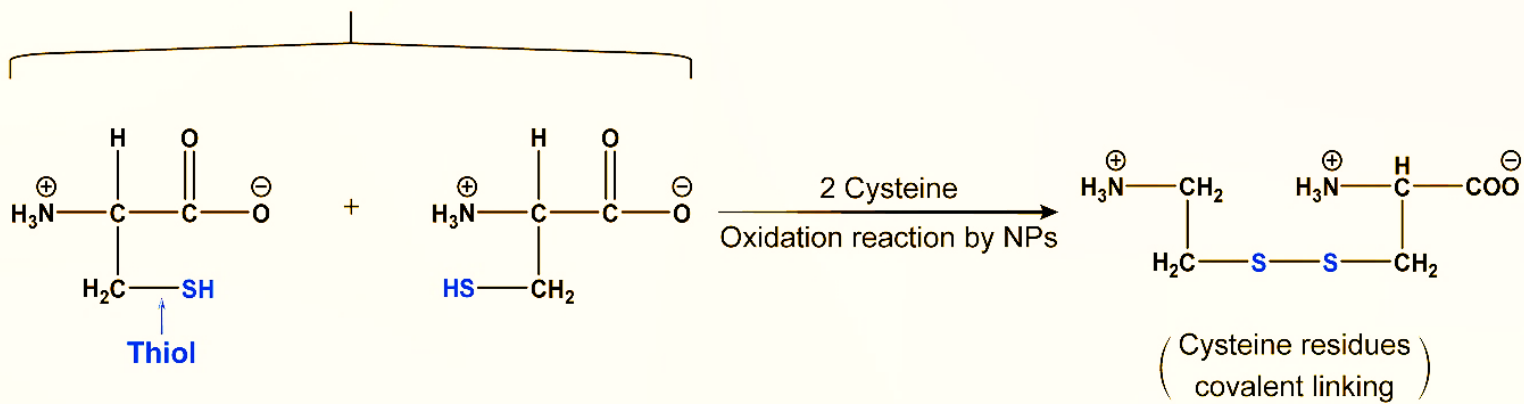

Figure 1. Strong NPs as an oxidant nanoagents are able to oxidize (to gain electron) sulfidryl groups or thiols $(\mathrm{R}-\mathrm{SH})$ and produce disulfide $(\mathrm{R}-\mathrm{S}-\mathrm{S}-\mathrm{R})$ bonds in biological systems. However, covalent disulfide bonds can be reduced (cleaves) by a reducing agent (such as a virus as a reductant) and generates two radicals of thiols and denatures a protein. This oxidation-reduction reaction can be cyclic and switchable.

\section{(c): To automatically "mirror" the strategy executed by the virus.}

The final nanofluid drug must have two very individual properties of nano-oxidant, and nanoreductant NPs together in a polar solution. Because, they should impart both important oxidation and reduction roles against the complex virus. When the virus attack to the vital cross folding proteins in DNA, they break disulfide $-\mathrm{S}-\mathrm{S}$ - bond to thiol $-\mathrm{RS}^{0}[8,9,10] . \mathrm{S}^{0}$ refers to (free radical). As a mirror strategy, smart reducing NPs (such as CNT-COOH) attack to a virus that has nested in a safe place in the human body and give it an electron, and at the end of the process, the disulfide 
bonds inside virus' protein coating would be broken. That is, indeed, the same problem that the destructive virus causes in the human body (a reduction state) and disrupts the immune system, and now is precisely imitated by novel powerful NPs (that carry reductant agents), against an uninvited guest (virus). Like a "mirror trading strategy" that allows us to copy the function of experienced and successful virus (as a natural system) and implement the same trades, in almost real time. Despite this tactic, the work of nanodrug is still remained; i.e. in-situ chemical oxidation/ engineering issue by releasing electrons [11]. Herein, oxidant NPs (such as $\mathrm{ZnO} \& \mathrm{Fe}_{3} \mathrm{O}_{4}$ ) play an effective role in oxidizing/repairing major damaged bonds in body that causes by reductant virus, repairing thiols to disulfide bonds. Therewith, the disulfide bonds are produced again and this is a thermodynamically favored process. Then, the reaction of virus and our smart NPs against each other is only a simple-complex chemical reaction of oxidation and reduction. The NPs should be energetic, fine sized, stable and not decompose during the redox reaction. This principle should be followed to fight with the viruses of AIDS, SARS and influenza, as well.

\section{(d) The extensive mutations create a very problematic state.}

Inside the gene of this virus 345 type of mutations have been reported, and new complex mutations are still on the way. Such virus mutations could be accompanied by genetic code mutations in human (such as changes in the amino acids of vital proteins or conversion of thiamine (vitamin B1) to guanine (one of the four nucleobases in the nucleic acid of DNA) [12] which lead to a pathogenicity even much higher than influenza. By changing the amino acid key, patients become more prone to inflammation and pulmonary attacks and bleeding. These symptoms indicate that we are dealing with a virus that is constantly changing and each time shows itself in a new way.

\section{(e) Drug nanoformulation development against coronavirus.}

In our proposed nanoformulation, the $\mathrm{ZnO}$ quantum dot nanoparticles ( $\mathrm{ZnO} Q$-Dots NPs) are extremely fine, very energetic, and a strong oxidizer (zinc metal can change from $2^{+}$to $4^{+}$in an ionic condition). Such Q-Dots employed to form disulfides covalent bonds, by producing reactive oxygen species (ROS), and support the protein folding process. $\mathrm{Fe}_{3} \mathrm{O}_{4} \mathrm{NPs}$ is also an assistant agent for the $\mathrm{ZnO}$ Q-Dots NPs, where $\mathrm{Fe}_{3} \mathrm{O}_{4}$ can attack in parallel with $\mathrm{ZnO}$ NPs or can support the $\mathrm{ZnO}$ NPs attacks [13]. The actual mechanism of action related to the presence of different nanoparticles in a nanofluid is still unknown. These two sets consist the oxidant part of the nanoformulation drug. On the other hand, attacking to the virus membrane, and destroying the virus' DNA structure, can also be found in CuO NPs researches [14,15].

The reduction part of our formulation is in charge with CNTs, which has been modified by a fatty acidic agent. The surface modified CNTs with carboxylic acid is the best option as a reducer agent, especially if the CNTs diameter is such small to be in range of 10-15 nm.

Remarkably, the proposed redox theory can also be true in relation to cancer cells that originate from virus. Also, the NPs can combat with bacteria and fungi during a simple chemical redox 
reaction, as well. Hence, the term of bombardment of virus by NPs is completely wrong. If the NPs with very small sizes, such as quantum dots $[16,17]$, could be able to penetrate into host cell membranes of virus/bacteria/fungi $[18,19,20]$ and create a hydrogen bonding with the cell membrane's proteins, they will destroy the structural bonds through oxidation-reduction communications and also inhibit post-attachment virus replications. However, we add that individual NPs should necessarily be able to form and produce reactive oxygen species (ROS) factors as well, like semiconductor $\mathrm{TiO}_{2}$, and $\mathrm{ZnO}$ NPs $[21,22]$. Based on our multiple experiences and findings, $\mathrm{Ag}$, Sn, and $\mathrm{Cu}$ may produce toxicity against human body, therefore it is proposed to do not use these metals alone. Therefore, we suggest that, these metals should be applied as NPs or as a modifying agent over the powerful CNTs NPs, graphene oxide (GO), and other safe hybrid nanomaterials and biocompatible nanopolymers [22]. With this strategy (while using water-based solutions) such synthesized nanocomposites would not be toxic anymore for human body [22]. This significant proposal can be applied for the development of vaccines and treatments against HIV/AIDS, and some subtypes of influenza virus and COVID-19.

When NPs are capable of establishing the electrons and free radicals, they can perform redox. why? Because: 1- in chemical oxidation reaction, the NPs must take electrons and oxide the opposite side agent and 2 - in reduction reaction must donate the electrons and reduce the opposite side component. Such displacement of electrons is responsible for the energetic nanoparticles.

It may be a question that if the AIDS virus or COVID-19 are reducer agents, since they have a protein cover, then, they should reduce the disulfide bonds of themselves (which means they act against themselves)? In response, it should be said that in laws of chemistry this theory is basically not true. There is no example of an oxidizing or reducing agent that perform these properties against themselves. If there is an oxidizer, it oxidizes the opposite side component, and not itself. Therefore, the COVID-19 virus, never destroys its disulfide protein bonds. This means that it does not reduce itself.

Attacking the ions of $\mathrm{CuO}$ NPs to the COVID -19 virus's membrane, and destroying the virus' DNA structure can be also supportive findings for NP's antiviral effects [14,15].

\section{Conclusions and future perspectives.}

Lessons can be learned from examining the redox reactions initiated and conducted by coronavirus as a type of natural systems. As a responsive strategy, we synthesized NPs, modified their surfaces to be either effective oxidizer or reductant, and formulated as a nanofluid as a reactive anti-covid-19. The first step to achieve an effective nanodrug is to determine the toxicity of the drug and detect the lowest and highest doses for the animal model. With the similar chemical predictions of this short manuscript but in solid tumors, as a very successful report for breast cancer cell line (MCF-7), we performed MTT assay test, the doses were determined and the IC50 were evaluated in different concentrations and at three times. For animal models and in vivo tests, IC50 were evaluated as $62.1,57.4$ and $55.7 \mu \mathrm{g} / \mathrm{ml}$ for 24,48 , and $72 \mathrm{~h}$ and the range of doses were 5-50 $\mu \mathrm{g} / \mathrm{ml}$. With this background, our synthesized anti-COVID -19 is currently being tested on human tissues, which can be applied to treat volunteer patients once the results are available. 


\section{Disclosure statement}

The authors report no conflicts of interest and are responsible for the content and writing of the manuscript.

\section{REFERENCES}

[1] C. Liu, Q. Zhou, Y. Li, L.V. Garner, S.P. Watkins, L. J. Carter, J. Smoot, A. C. Gregg, A. D. Daniels, S. Jervey, D. Albaiu, "Research and Development on Therapeutic Agents and Vaccines for COVID-19 and Related Human Coronavirus Diseases", ACS Cent. Sci., Vol. 6, No. 3, 2020, pp. 315-331.

[2] M. T. ul Qamar, S.M. Alqahtani, M.A. Alamri, L. L Chen, "Structural basis of SARS-CoV-2 3CLpro and anti-COVID-19 drug discovery from medicinal plants", Journal of Pharmaceutical Analysis, Available online 26 March 2020, https://doi.org/10.1016/j.jpha.2020.03.009

[3] B. Robson, "COVID-19 Coronavirus spike protein analysis for synthetic vaccines, a peptidomimetic antagonist, and therapeutic drugs, and analysis of a proposed achilles' heel conserved region to minimize probability of escape mutations and drug resistance" Comput Biol Med. Vol. 121, 2020, pp. 103749. doi: 10.1016/j.compbiomed.2020.103749

[4] P. Yang, X. Wang, "COVID-19: a new challenge for human beings" Cellular \& Molecular Immunology, Vol. 17, 2020, pp. 555-557. Open Access.

[5] H, Huang, S.C. Harrison, G. Verdin, "Trapping of a catalytic HIV reverse transcriptase ·template: primer complex through a disulfide bond", Chemistry and Biology, Vol. 7, No. 5, 2000, pp. 355-364. https://doi.org/10.1016/S1074-5521(00)00113-7

[6] S. Hati, S. Bhattacharyya, "Impact of Thiol-Disulfide Balance on the Binding of Covid-19 Spike Protein with Angiotensin-Converting Enzyme 2 Receptor”, ACS Omega, 2020, https://doi.org/10.1021/acsomega.0c02125

[7] J.C. Lukesh, M.J. Palte, R.T. Raines," A Potent, Versatile Disulfide-Reducing Agent from Aspartic Acid", J. Am. Chem. Soc., Vol. 134, No. 9, 2012, pp.4057-4059. https://doi.org/10.1021/ja211931f

[8] D. Fass, C. Thorpe, "Chemistry and Enzymology of Disulfide Cross-linking in Proteins”, Chem Rev., Vol. 118, No 3, 2018, pp. 1169-1198. doi: 10.1021/acs.chemrev.7b00123

[9] M.V. Trivedi, J.S. Laurence, T.J. Siahaan, "The role of thiols and disulfides in protein chemical and physical stability”, Curr Protein Pept Sci., Vol.10, No. 6, 2009, pp. 614-625.

[10] A. de Marco, Strategies for successful recombinant expression of disulfide bond-dependent proteins in Escherichia coli, "Microbial Cell Factories, Vol. 8, No. 26, 2009, pp. 1-18. DOI: 10.1186/1475-2859-8-26 
[11] R. O Young, "Chlorine Dioxide $\left(\mathrm{CLO}_{2}\right)$ as a Non-Toxic Antimicrobial Agent for Virus, Bacteria and Yeast (Candida Albicans)", International Journal of Vaccines and Vaccination, Vol. 2 No. 6, 2016, pp.1-12.

[12] S. Dhir, Tarasenko, Napoli, C. Giuliv, "Neurological, Psychiatric, and Biochemical Aspects of Thiamine Deficiency in Children and Adults" Front Psychiatry, Vol. 10, No. 207, 2019, pp. 115. doi: 10.3389/fpsyt.2019.00207

[13] A.M. Yousefi, A. Safaroghli-Azar, Z. Fakhroueian, D. Bashash, “ $\mathrm{ZnO} / \mathrm{CNT} @ \mathrm{Fe}_{3} \mathrm{O}_{4}$ induces ROS-mediated apoptosis in chronic myeloid leukemia (CML) cells: an emerging prospective for nanoparticles in leukemia treatment", Artificial Cells, Nanomedicine, and Biotechnology, Vol. 48, No. 1, 2020, pp. 735-745. https://doi.org/10.1080/21691401.2020.1748885

[14] K. Sunada, M. Minoshima, K. Hashimoto, "Highly efficient antiviral and antibacterial activities of solid-state cuprous compounds", Journal of hazardous materials, Vol. 235-236, 2012, pp.265-270. DOI: 10.1016/j.jhazmat.2012.07.052

[15] J. Zhou, Z. Hu, F. Zabihi, Z. Chen, M. Zhu, "Progress and Perspective of Antiviral Protective Material", Advanced Fiber Materials, Vol. 2, 2020, pp.123-139. https://doi.org/10.1007/s42765$\underline{020-00047-7}$

[16] Z. Fakhroueian, A. Mozafari Dehshiri, F. Katouzian, P. Esmaeilzadeh, "In vitro cytotoxic effects of modified zinc oxide quantum dots on breast cancer cell lines (MCF7), colon cancer cell lines (HT29) and various fungi”, Journal of Nanoparticles Research, Vol. 16: 2483, 2014, pp.114.

[17] Z. Fakhroueian, R. Vahabpour, M. Assmar, A. Massiha, A. Zahedi, P. Esmaeilzadeh, F. Katouzian, S. Rezaei, P. Keyhanvar, A. Mozafari Dehshiri, "ZnO Q-dots as a potent therapeutic nanomedicine for in vitro cytotoxicity evaluation of mouth KB44, breast MCF7, colon HT29 and HeLa cancer cell lines, mouse ear swelling tests in vivo and its side effects using the animal model", Artificial Cells, Nanomedicine, and Biotechnology, 2018, pp.1-17. https://doi.org/10.1080/21691401.2018.1452023

[18] Z. Fakhroueian, F.M. Harsini, F. Chalabian, F. Katouzian, A. Shafiekhani, P. Esmaeilzadeh, "Influence of Modified ZnO Quantum Dots and Nanostructures as New Antibacterials", Advances in Nanoparticles, Vol. 2, 2013, pp. 247-258. doi:10.4236/anp.2013.23035

[19] F. Katouzian, Z. Fakhroueian, S. Moradi Bidhendi, "The Interesting of Antifungal Effects of Novel In Vitro Fabrics of Stabilized ZnO Nanofluids", Advances in Nanoparticles, Vol. 5, 2016, pp. 206-223. http://www.scirp.org/journal/anp

[20] Z. Fakhroueian, F, Katouzian, P. Esmaeilzadeh, S. Moradi Bidhendi, P. Esmaeilzadeh, "Enhanced engineered $\mathrm{ZnO}$ nanostructures and their antibacterial activity against urinary, gastrointestinal, respiratory and dermal genital infections", Applied Nanoscience, Vol. 9, 2019, pp. 1759-1773. https://doi.org/10.1007/s13204-019-00996-5 
[21] J. Jiang, J. Pi, J. Cai, "The Advancing of Zinc Oxide Nanoparticles for Biomedical Applications", Bioinorganic Chemistry and Applications, Vol. 2018, pp.1-18. https://doi.org/10.1155/2018/1062562

[22] M. Chiara Sportelli, M. Izzi, E. A. Kukushkina, S. I. Hossain, R. A. Picca, N. Ditaranto N. Cioffi, "Can Nanotechnology and Materials Science Help the Fight against SARS-CoV-2?", Nanomaterials, Vol. 10, No. 802, 2020, pp. 1-12. doi:10.3390/nano10040802 